\title{
Glycérol : état du marché et débouchés potentiels - hiver 2006
}

\author{
Michael HEMING ${ }^{1}$ \\ Sylvain CLAUDE ${ }^{2}$ \\ ${ }^{1} \mathrm{HB}$ International SAS, 26 bis rue de l'Ermitage, \\ BP 80031, 95161 Montmorency Cedex \\ $<$ mike.heming@hbint.com> \\ ${ }^{2}$ ONIDOL/SOFIPROTEOL \\ 12 avenue George $\mathrm{V}$ \\ 75008 Paris \\ <s.claude@prolea.com>
}

\begin{abstract}
Glycerine is a by-product of the biodiesel industry. Owing to the exponential growth of biodiesel production worldwide and particularly in Europe, the glycerine market has been driven during the last few years by the oversupply being generated by the biodiesel industry. Prices of both refined and crude glycerine have fallen to all-time lows. Consequently, there are clear signs that demand for glycerine is growing strongly with new usages both for crude and refined glycerine now to the fore. This article attempts to analyse to what extent a "substitution/new-usage breakthrough » for glycerine is occurring and will propose a supply/demand scenario for the coming 3 years. It will also attempt to predict how glycerine prices will develop over the same period.
\end{abstract}

Key words: biodiesel, glycerine, glycerol, market, supply, demand, prices, new usages

\section{Introduction}

«En fait, dans l'histoire de la glycérine, on a pu assister souvent à des baisses considérables sans que la production en ait été en rien diminuée. De même, on a pu voir les prix doubler, tripler ou quadrupler sans observer de diminution de consommation » (Société française des glycérines en 1933/34, Fehrenbach imprimeur, Paris).

Tous les acteurs et observateurs du marché de la glycérine s'accordent sur ce point : les fluctuations de ses cours n'ont pas cessé et demeurent spectaculaires depuis cent trente ans. Le premier usage industriel de la glycérine remonte à 1862 et s'adressa à l'industrie textile. Ce résultat permit aux fabricants de bougies stéariques, producteurs involontaires de glycérine, d'écouler avec profit un sous-produit jeté au ruisseau. Dix ans plus tard, les nombreux emplois qui furent successivement trouvés, prônés et adoptés absorbaient toute la glycérine produite par l'industrie stéarique, environ 10000 tonnes/an. La première spéculation sur la glycérine eut lieu en 1872 suite aux promesses de développement de la dynamite : les prix doublèrent mais les prévisions de consommation ne furent pas au rendez-vous ; les cours chutèrent.

\section{État des lieux}

À la question : «Qu'en est-il en 2006 ? ", I'on peut répondre qu'actuellement la notion d'équilibre entre l'offre et la demande a perdu son sens: toute la glycérine du marché est désormais d'origine naturelle ; elle est redevenue en quelque sorte un produit « fatal ». Heureusement, l'histoire montre que les cours se sont toujours relevés. Un premier régulateur réside dans la production de glycérine synthétique et si, en 1985, $25 \%$ de la production était d'origine synthétique puis $10 \%$ en 1995 et $2 \%$ en 2005 , elle est dorénavant pratiquement inexistante ; DOW a stoppé la production du site de Freeport le 31 janvier 2006.

Tableau 1. Sources de production mondiale de glycérine 1992-2008.

\begin{tabular}{|c|c|c|c|c|c|c|c|}
\hline '000 mt 100\% & $1992^{\mathrm{a}}$ & $1995^{b}$ & $1998^{\mathrm{C}}$ & $1999^{d}$ & $2003^{e}$ & $2005^{f}$ & $2008^{g}$ \\
\hline Savons & 208 & 210 & 199 & 198 & 180 & 160 & 130 \\
\hline Acides gras & 268 & 286 & 304 & 322 & 350 & 410 & 480 \\
\hline Biodiesel & 6 & 40 & 55 & 57 & 160 & 270 & 600 \\
\hline Alcools gras & 78 & 99 & 109 & 108 & 110 & 130 & 220 \\
\hline Synthétique & 78 & 80 & 95 & 75 & 80 & 20 & - \\
\hline \multicolumn{8}{|l|}{ Autres : } \\
\hline Esters méthyliques & & 15 & 20 & 24 & 25 & 30 & 30 \\
\hline « Fat Substitutes» & & & 3 & 5 & 5 & 5 & 5 \\
\hline Fermentation & & & 15 & 15 & 20 & 10 & 10 \\
\hline TOTAL & 638 & 730 & 800 & 804 & 930 & 1035 & 1475 \\
\hline \multirow{2}{*}{$\begin{array}{l}\text { Taux annuel } \\
\text { de croissance }\end{array}$} & & $4,5 \%$ & $3 \%$ & $0,5 \%$ & $3,75 \%$ & $5,5 \%$ & $12,5 \%$ \\
\hline & & & & $3,75 \%$ & & & \\
\hline
\end{tabular}

a $1^{\text {st }}$ World Glycerine Conference, HBI, 1993.

b $2^{\text {nd }}$ World Glycerine Conference, $\mathrm{HBI}, 1996$.

c Données présentées par HBI au Glycerine Workshop organisé la Commission Européenne, DGXII.

d Présenté par HBI au WCO à Kuala Lumpur, septembre 2000.

e Estimations HBI, publiées dans Gunstove F, Heming M. Glycerol-an important product of the oleochemical industry. Lipid Technology $2004 ; 16(8)$.

${ }^{f}$ Estimation $\mathrm{HBI}$.

g Estimation $\mathrm{HBI}$
La production mondiale de glycérine était de $730000 \mathrm{t}$ en 1995 , elle a dépassé $1 \mathrm{Mt}$ en 2005 et devrait atteindre 1,5 Mt en 2008, (tableau 1). Si le taux de croissance de l'offre a été modéré jusqu'en 2005, environ 3,75 \%/an, il atteindra vraisemblablement 12,5\%/an entre 2005 et 2008.

Si les usages traditionnels ne se développent pas ou s'il n'en apparaît pas de nouveaux, 


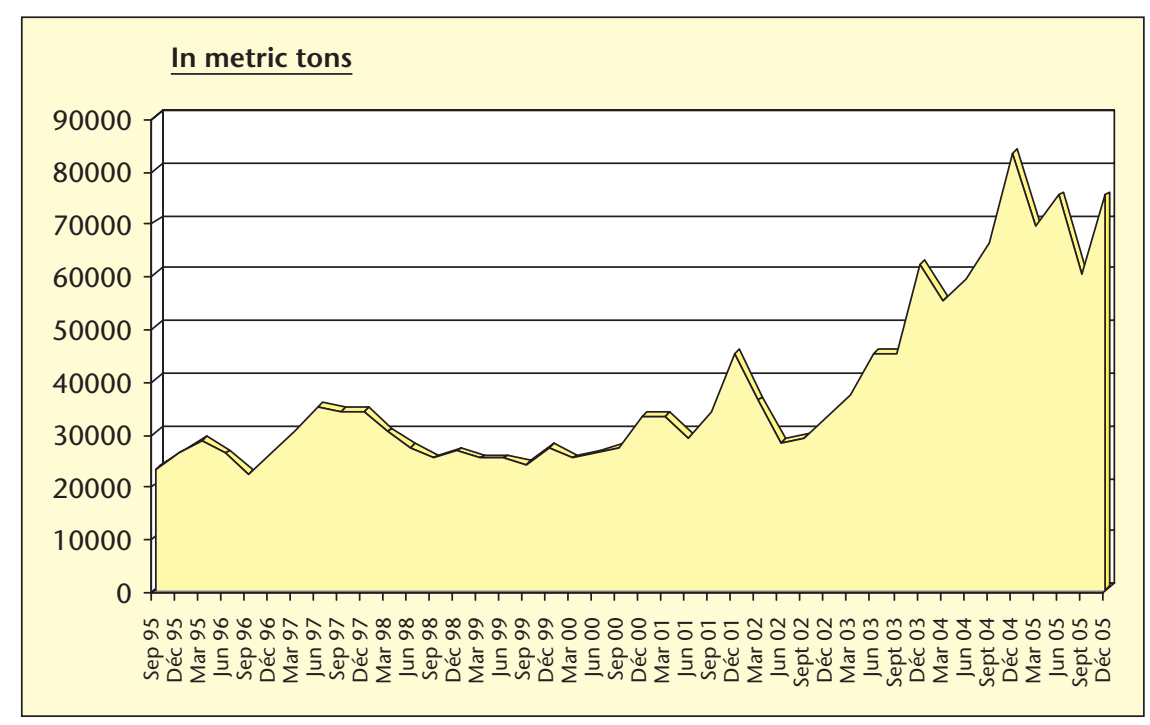

Figure 1. Stocks de glycérine des producteurs européens 1995-2005.

Source: $H B I$.

I'offre risque de dépasser la demande en 2008 d'environ 400000 tonnes. Aussi la question se posera-elle de savoir comment la demande pourra la compenser.

Jusqu'en 2005 la demande arrivait tant bien que mal à équilibrer l'offre, notamment grâce à la constitution de stocks importants: 75000 tonnes en « brute et raffinée » fin 2005 (figure 1).

Au cours de cette année 2006, l'équilibre précaire ainsi maintenu risque de se rompre. Néanmoins et contre toute attente, il pourrait se rétablir à la mi-2008 ou même avant. II y a en effet des signes avant-coureurs d'une croissance accrue de la demande, autant pour la glycérine raffinée que pour la brute.

\section{Quid des prix ?}

L'absence de parallélisme décrit plus haut entre production et consommation de glycérine s'est concrétisée de façon simple et «brutale » : la glycérine raffinée se vend au tiers du prix qu'elle atteignait en 1995 (figure 2 et tableau 2), quant à la glycérine brute, elle a perdu $90 \%$ de sa valeur (figure 3 et tableau 3). La chute récente du cours de la qualité brute (elle vaut moins de $10 \%$ de son prix de 1995) est plus marquée que pour la qualité raffinée. Cela peut s'expliquer par l'insuffisance des capacités de raffinage et favorise son exportation à bas prix tout en encourageant la découverte de débouchés se contentant de cette qualité. La glycérine brute actuellement commercialisée provient désormais du biodiesel et non plus de la savonnerie.

En 2005, les USA montrent une production glycérol en déclin, une dépendance accrue des importations ( $60 \%$ de la consommation) et une demande en baisse depuis 2000 (figure 4 et Source: $H B I$. tableau 4). Ce déclin de la production s'explique par un ralentissement des activités en savonnerie, en oléochimie et par l'arrêt progressif puis total de la production de glycérine synthétique.

La situation semblerait évoluer dans le même sens que l'Europe, avec l'apparition d'unités de biodiesel et des taux d'incorporation diversement financés selon les états (tableau 5). Plus important, I'obligation de désulfurer le diesel (200 M tonnes/an) : $1 \%$ d'incorporation de biodiesel - pour compenser le pouvoir lubrifiant apporté par le soufre - entraînerait la production de 200000 tonnes de glycérine, soit un peu plus que la consommation intérieure. En 2005, 50 \% du biodiesel américain a été exporté vers l'Allemagne, en 2006 cela devrait représenter $30 \%$.

\section{L'Europe}

La situation européenne contraste avec celle des USA. La production, la consommation et les exports de glycérine ont pratiquement doublé en Europe depuis 1995, pour atteindre 385000 tonnes en 2005 (tableau 6). On observe que production et consommation sont équilibrées et que les imports demeurent faibles sur la durée (figure 5).

\section{Évolutions}

L'activité biodiesel, presque exclusivement européenne (France, Allemagne, Italie), est à I'origine de ces évolutions : $57 \%$ de la glycérine produite en Europe provient de cette activité. Les 385000 tonnes produites au total en

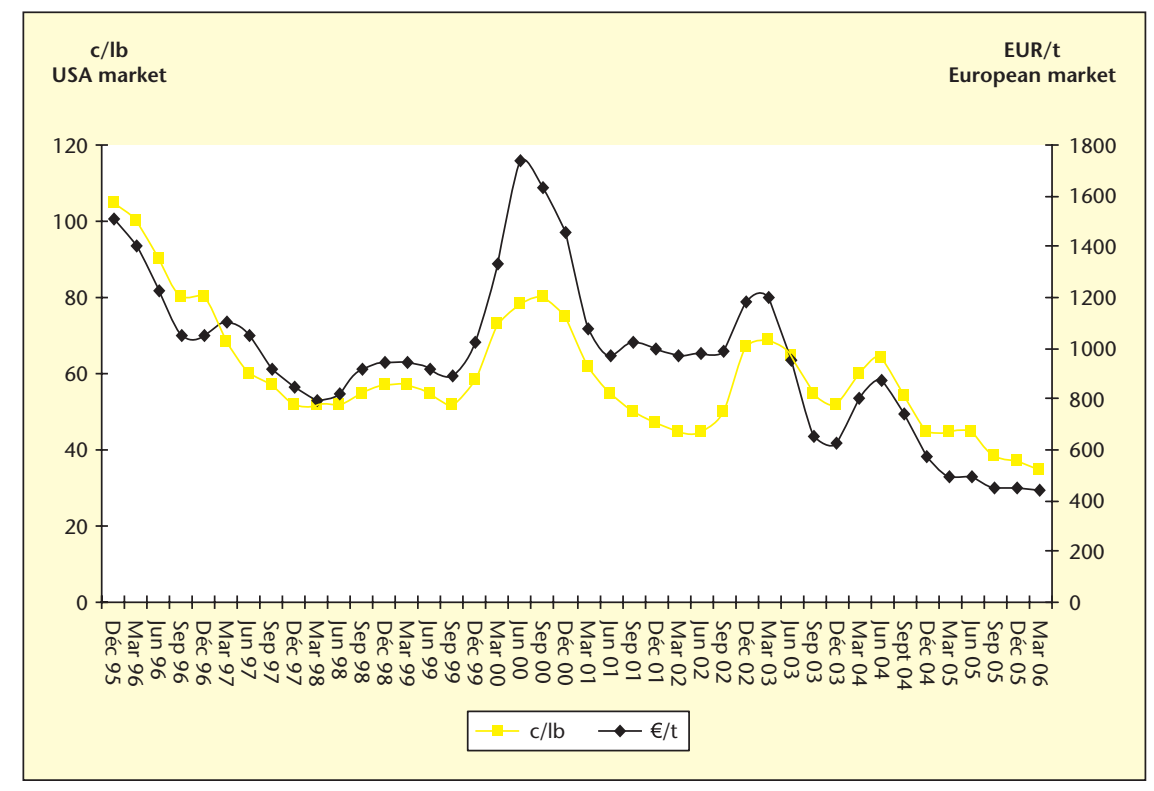

Figure 2. Prix «spot » «vrac-rendu » aux USA et en Europe pour la glycerine kasher 99,7\% 1995-2006. 
Tableau 2. Comparaison des prix « spot » pour la glycérine raffinée « kasher » entre les USA et L'Europe, 1995-2006.

\begin{tabular}{|c|c|c|c|c|}
\hline \multirow[t]{2}{*}{ Date } & \multicolumn{2}{|c|}{ USA Qualité kasher } & \multirow{2}{*}{$\begin{array}{c}\text { Europe Qualité kasher } \\
€ / \mathbf{T}\end{array}$} & \multirow{2}{*}{$\begin{array}{c}\text { « Premium » } \\
€ / T\end{array}$} \\
\hline & $\mathbf{c} / \mathbf{l b}$ & $€ / \mathbf{T}$ & & \\
\hline Décembre 1995 & 105 & 1715 & 1508 & 207 \\
\hline Décembre 1996 & 80 & 1406 & 1048 & 358 \\
\hline Décembre 1997 & 52 & 1054 & 844 & 210 \\
\hline Décembre 1998 & 57 & 1092 & 946 & 146 \\
\hline Décembre 1999 & 58 & 1274 & 1023 & 251 \\
\hline Décembre 2000 & 75 & 1876 & 1457 & 419 \\
\hline Décembre 2001 & 47 & 1165 & 997 & 168 \\
\hline Décembre 2002 & 67 & 1477 & 1180 & 297 \\
\hline Mars 2003 & 69 & 1421 & 1200 & 221 \\
\hline Juin 2003 & 65 & 1224 & 950 & 274 \\
\hline Septembre 2003 & 55 & 1102 & 650 & 452 \\
\hline Décembre 2003 & 52 & 932 & 630 & 302 \\
\hline Mars 2004 & 60 & 1075 & 800 & 275 \\
\hline Juin 2004 & 64 & 1175 & 870 & 305 \\
\hline Septembre 2004 & 54 & 992 & 740 & 252 \\
\hline Décembre 2004 & 45 & 745 & 570 & 175 \\
\hline Mars 2005 & 45 & 751 & 490 & 261 \\
\hline Juin 2005 & 45 & 826 & 490 & 336 \\
\hline Septembre 2005 & 38 & 675 & 450 & 225 \\
\hline Decembre 2005 & 37 & 697 & 450 & 247 \\
\hline Mars 2006 & 35 & 648 & 440 & 208 \\
\hline
\end{tabular}

Source : HBI.

Moyenne des prix « spot » « vrac-rendu ». Taux de change: milieu du mois de cotation ; pour mars 2006, le taux de change utilisé fut : 1 EUR = 1,19 USD.

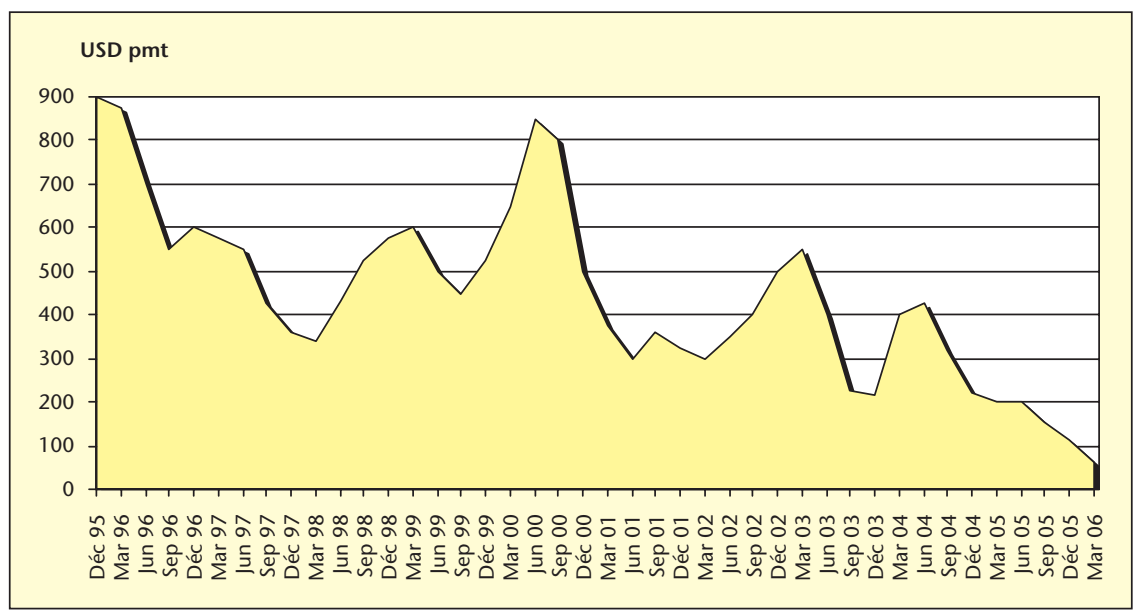

Figure 3. Évolution du prix de la glycerine brute $80 \% *$ sur le marché européen, 1995-2005, en USD pmt. Source: HBI. Prix d'un grade moyen de glycérine brute base $80 \%$, CFR Rotterdam, jusqu'à juin 2005, et depuis, moyenne des cotations FCA départ Europe. Puisque le coût de transport depuis Rotterdam vers un raffineur de glycérine est approximativement le même que depuis un site de production de biodiesel, la comparaison est valide. Taux de change utilisé pour mars 2006:1 EUR =1,19 USD.

2005 sont à comparer à une demande qui s'est élevée à 351000 tonnes. Entre 2004 et 2005, la demande a crû de $4,2 \%$ (PNB $=1,4 \%$ en Europe sur cette période). Le taux de crois- sance moyen de la demande s'élève à 5,75\% sur la période 1997/2005. L'Europe reste fortement exportatrice de glycérine raffinée vers les USA.

\section{Perspectives}

L'offre

À I'horizon 2007, une production de 250000 tonnes supplémentaires de glycérine ex-biodiesel est à prévoir en Europe. À I'horizon 2007-2008 et à l'échelle du monde, si on considère les prévisions de production en biodiesel, il faut s'attendre à la mise sur le marché de 400000 tonnes supplémentaire de glycérine par rapport à 2005 (tableaux 1et 7).

Concernant ces futurs approvisionnements, les principaux éléments à prendre en compte pour la période 2005-2008 sont:

1. Les grands savonniers abandonnent désormais la glycérine dans le savon ("Swing process »). II y a dix ans, $30 \%$ de la glycérine provenait des savonneries. Ce chiffre est tombé à $10 \%$.

2. La demande en savon des pays développés baisse globalement. Lorsque localement on observe une augmentation de consommation, les bondillons livrés sont obtenus à partir d'acides gras.

3. La demande en acides gras augmente d'environ $5 \% / a n$.

4. La production de 600000 tonnes d'alcools gras supplémentaires en 2008 est très probable, en conséquence directe de l'augmentation du prix de l'éthylène et des restrictions environnementales sur les LABS.

\section{La demande}

Avec un taux de croissance de $3,75 \%$ la demande en glycérol pour les usages établis évoluera ainsi (kt) :

Glycérol $2005 \quad 2006 \quad 2007 \quad 2008$ Demande $1035 \quad 1208 \quad 1417 \quad 1667$

Pour que le marché reste équilibré à I'horizon 2008 (tableau 1), la demande en glycérine devra croître de 12,5\%/an, à comparer avec les 3,75\% observés entre 1992 et 2005 . Si les usages traditionnels ne se développent pas ou si de nouveaux n'apparaissent pas, l'offre risque de dépasser la demande d'environ 400000 tonnes en 2008. Aussi le problème se pose de savoir comment la demande peut la compenser. L'apparition de nouveaux usages, aux côtés des utilisations traditionnelles, pourrait le résoudre.

Voici quelques exemples en vrac que nous avons identifiés dans un premier temps :

- Substitution des polyols : ils sont au nombre de 5 : le sorbitol, l'éthylène glycol, le propylène glycol, le pentaérythritol, le triméthylolpropane.

- Sorbitol: le marché mondial du sorbitol s'élève à $1 \mathrm{M}$ tonnes. La moitié en revient à la société Roquette. $75 \%$ du sorbitol est substi- 
Tableau 3. Prix de la glycérine brute 80 \% exprimé en pourcentage du prix de la qualité raffinée, grade pharmaceutique, en Europe, 1995-2006.

\begin{tabular}{|c|c|c|}
\hline Date & $\begin{array}{c}\text { Glycérine brute } 80 \% \text {, vrac, } \\
\text { USD pmt }\end{array}$ & $\begin{array}{c}\text { Prix de la glycérine brute } 80 \% \\
\text { en pourcentage du prix de la qualité } \\
\text { pharmaceutique en Europe }\end{array}$ \\
\hline Décembre 1995 & 900 & $44 \%$ \\
\hline Décembre 1996 & 600 & $46 \%$ \\
\hline Décembre 1997 & 360 & $39 \%$ \\
\hline Décembre 1998 & 575 & $53 \%$ \\
\hline Décembre 1999 & 525 & $51 \%$ \\
\hline Décembre 2000 & 500 & $39 \%$ \\
\hline Décembre 2001 & 325 & $37 \%$ \\
\hline Mars 2002 & 300 & $42 \%$ \\
\hline Juin 2002 & 350 & $45 \%$ \\
\hline Septembre 2002 & 400 & $48 \%$ \\
\hline Décembre 2002 & 500 & $50 \%$ \\
\hline Mars 2003 & 550 & $38 \%$ \\
\hline Juin 2003 & 400 & $35 \%$ \\
\hline Septembre 2003 & 225 & $28 \%$ \\
\hline Décembre 2003 & 215 & $28 \%$ \\
\hline Mars 2004 & 400 & $40 \%$ \\
\hline Juin 2004 & 425 & $40 \%$ \\
\hline Septembre 2004 & 320 & $36 \%$ \\
\hline Décembre 2004 & 220 & $29 \%$ \\
\hline Mars 2005 & 200 & $31 \%$ \\
\hline Juin 2005 & 200 & $34 \%$ \\
\hline Septembre 2005 & 155 & $28 \%$ \\
\hline Décembre 2005 & 117 & $22 \%$ \\
\hline Mars 2006 & 60 & $14 \%$ \\
\hline
\end{tabular}

\section{Source : $\mathrm{HBI}$.}

Note : Jusqu'à juin 2005, moyenne des prix CFR Rotterdam et, depuis, prix FCA ex-unités de biodiesel. Taux de change : milieu du mois de cotation; pour mars 2006, le taux de change utilisé fut : 1 EUR =1,19 USD.

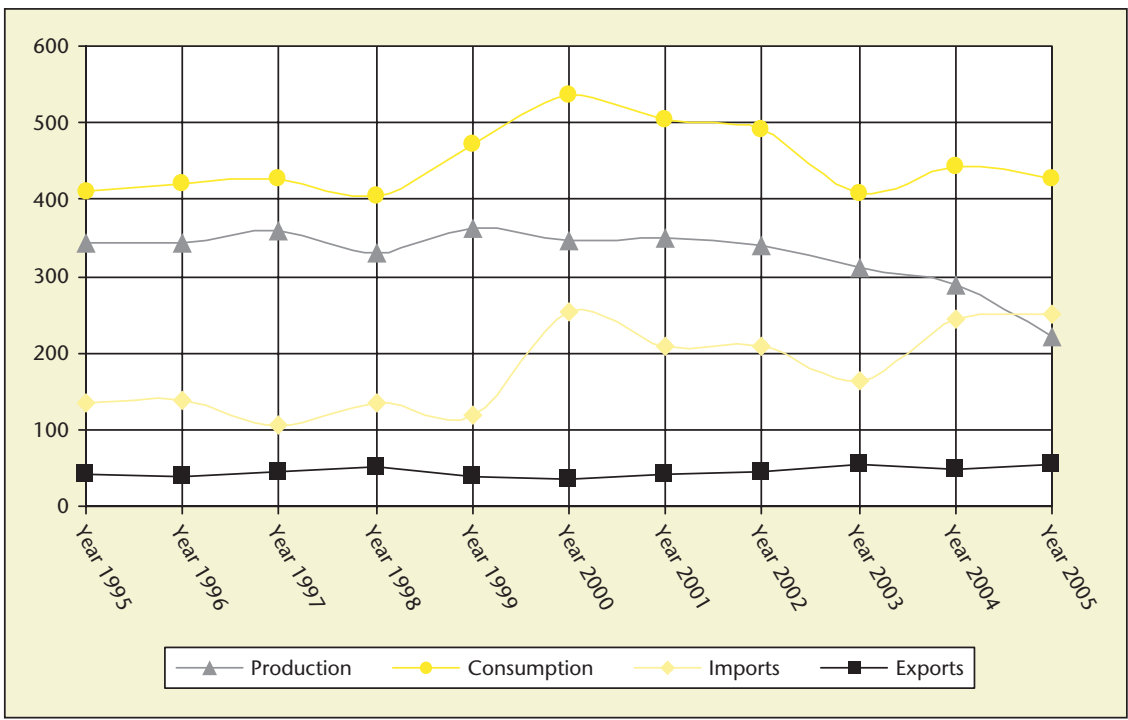

Figure 4. USA; production, consommation, imports et exports de glycérine, 1995-2005 (en million lbs) Source : US Department of Commerce (Via SDA).
Tableau 4. Production de glycérine aus USA 1995-2005 (en milliers de tonnes).

\begin{tabular}{|lcc|}
\hline \multirow{2}{*}{ Année } & \multicolumn{2}{c|}{ Production } \\
\cline { 2 - 3 } & Volume & Index \\
\hline 1995 & 155 & 100 \\
1996 & 150 & 97 \\
1997 & 163 & 105 \\
1998 & 149 & 96 \\
1999 & 164 & 105 \\
2000 & 156 & 101 \\
2001 & 159 & 102 \\
2002 & 154 & 99 \\
2003 & 141 & 91 \\
2004 & 131 & 85 \\
2005 & 100 & 64 \\
\hline
\end{tabular}

Source : US Department of Commerce (via SDA).

Tableau 5. Estimation de la production de biodiesel aux USA 2004-2010 en milliers de mt.

\begin{tabular}{|lc|}
\hline Année & Volume \\
\hline 2004 & 100 \\
2005 & 300 \\
2006 & 500 \\
2010 & 1500 \\
\hline
\end{tabular}

Source : USDA pour 2004. Estimations HBI pour les autres années.

tuable par le glycérol. $30 \%$ en masse d'une pâte dentifrice est constituée de glycérol ou de sorbitol. La consigne chez un major de I'hygiène corporelle est qu'au moins $30 \%$ du polyol soit du glycérol.

- On enregistre vers la Chine une augmentation des importations de glycérol : 60000 tonnes en 2004 puis 120000 tonnes en 2005. II est estimé qu'en 200630000 tonnes de cette glycérine serviront à substituer des polyols.

- Glycols : le principal débouché de l'éthylène glycol (MEG) est le PET, créneau guère facile à déloger. Quelques 10000 tonnes de MEG pour les produits lave-glaces, quelques centaines de milliers pour les antigels radiateurs: 200000 tonnes en « première monte » et un à deux remplissages totalisant de leur côté une quantité équivalente (Europe); soit 400500000 tonnes de potentiel (formule $=95 \%$ $\mathrm{MG}+2 \%$ eau $+3 \%$ additifs). "De-icing " aéronautique : deux applications différentes; le dégivrage des avions (préventif + curatif) et le « déverglaçage ». La première est très technique, il y a beaucoup d'homologations/ barrages à passer. La seconde représente 1000 t/an pour les ADP. Déjà utilisé sur des aérodromes et héliports aux USA. La crainte des formulateurs n'est pas d'ordre technique mais économique (Cf. fluctuations prix du glycérol). 
Tableau 6. Production européenne de glycérine 1995-2005 (en milliers de tonnes).

\begin{tabular}{|lcc|}
\hline Année & Volume & Index \\
\hline 1995 & 215 & 100 \\
1996 & 224 & 104 \\
1997 & 250 & 116 \\
1998 & 239 & 111 \\
1999 & 268 & 125 \\
2000 & 296 & 138 \\
2001 & 297 & 138 \\
2002 & 300 & 140 \\
2003 & 315 & 147 \\
2004 & 360 & 167 \\
2005 & 385 & 179 \\
\hline
\end{tabular}

Source : $\mathrm{HBI}$.

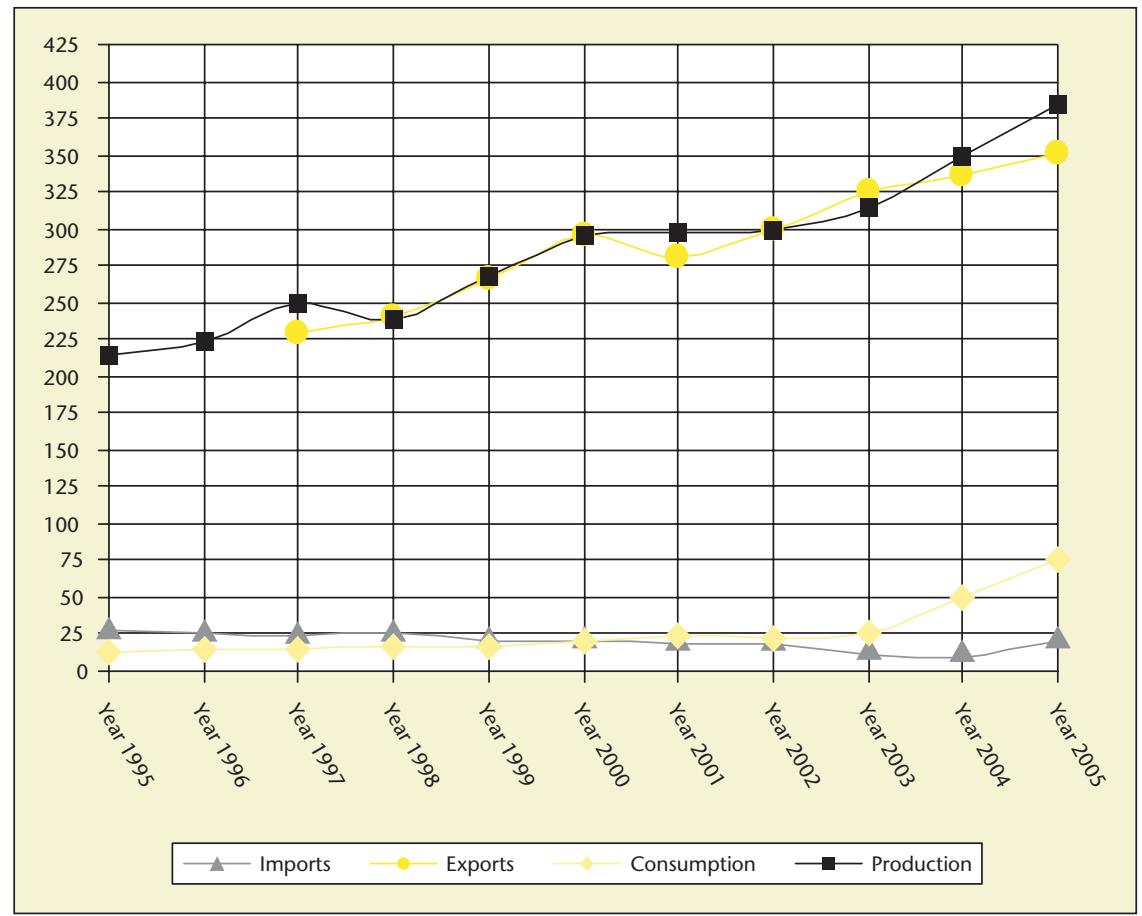

Figure 5. Europe, production, consommation en glycérine " ex continent », imports et exports 1995-2005

('000 mt).

Source : $H B I$

Tableau 7. Production mondiale de biodiesel en 2002-2004 et estimations jusqu'en 2007 (en milliers de tonnes).

\begin{tabular}{|c|c|c|c|c|c|c|c|c|c|c|c|c|}
\hline & 2002 & $\%$ & 2003 & $\%$ & 2004 & $\%$ & 2005 & $\%$ & 2006 & $\%$ & 2007 & $\%$ \\
\hline $\begin{array}{l}\text { Europe } \\
\text { dont }\end{array}$ & 1065 & 97 & 1434 & 96 & 1933 & 94 & 2200 & 81 & 3200 & 80 & 4700 & 81 \\
\hline Allemagne & 450 & 41 & 715 & 48 & 1035 & 50 & 1200 & 44 & 1800 & 45 & 2400 & 41 \\
\hline France & 366 & 33 & 357 & 24 & 348 & 17 & 450 & 17 & 600 & 15 & 1000 & 17 \\
\hline $\begin{array}{l}\text { Reste du } \\
\text { monde } \\
\text { dont }\end{array}$ & 35 & 3 & 65 & 4 & 120 & 6 & 500 & 19 & 800 & 20 & 1100 & 19 \\
\hline USA & 30 & 3 & 60 & 4 & 100 & 5 & 300 & 11 & 500 & 13 & 750 & 13 \\
\hline Total & 1100 & 100 & 1499 & 100 & 2053 & 100 & 2700 & 100 & 4000 & 100 & 5800 & 100 \\
\hline
\end{tabular}

Sources : European Biodiesel Board, USDA 2004, CCC Data, HBI. Note : les pourcentages sont arrondis.
- Propylène glycol : la conversion du glycérol en ce diol a été travaillée par ADM et IPCI. Les investissements nécessaires seraient énormes ; une affaire à suivre. Alimentation animale: le propylène glycol est incorporé dans la ration des vaches venant de mettre bas comme supplément énergétique. Cela représente au moins 5000 tonnes en France, qui sont à multiplier par 10 pour l'Europe et encore par 10 pour le monde ; soit un total de 0,5 M tonnes. Notre hypothèse de travail reposera sur une substitution de 10000 tonnes en 2006 pouvant doubler chaque année.

- Combustion : dans ce secteur, il faut se référer au mazout (fod) : 500\$/t, $\mathrm{PC}(\mathrm{I} / \mathrm{S})$ $40 \mathrm{MJ} / \mathrm{kg}$. Sous le rapport des pouvoirs calorifiques, la glycérine pure, $19 \mathrm{MJ} / \mathrm{kg}$, pourrait valoir $200 € /$ t. Comme elle brûle «moins bien» que le mazout, il faut prévoir un «malus » qui la dévalorise jusqu'à $100 € / t$. II faut noter que le glycérol ne fournit qu'un tiers de l'énergie obtenue avec une mélasse (100$120 € / \mathrm{t}$ ) et qu'une glycérine brute à $80 \%$ se vend de 80 à $100 € / t$ (rendu). Dans les faits, la glycérine brute commence à être brûlée et le sera au moins jusqu'en 2008. Pour la qualité brute, la présence de MONG (matières organiques non glycérineuses) et de méthanol améliore le pouvoir calorifique. Restrictions réglementaires à la combustion : quelques qualités de glycérine brute contiennent des chlorures (dioxines).

- L'épichlorhydrine: $1 \mathrm{M}$ tonnes en 2005 (800 kt en 2002, 900 kt en 2003). En 2003, environ $76 \%$ de la consommation était consacrée aux résines « époxy », $9 \%$ à la glycérine synthétique et le reste pour divers usages; élastomères, résines polyamides, tensioactifs, etc. Sur les cinq dernières années, croissance modeste aux USA : 2-2,5\% mais élevée en Asie : $7 \%$. Unilever a travaillé sur la conversion du glycérol en épichlorhydrine, il y a trente ans, alors que le propylène était à un prix qui semblerait compétitif aujourd'hui. Solvay prévoit la construction à Tavaux (Jura) d'une première unité de 10000 tonnes qui pourrait être dupliquée.

- Carbonate de glycérol: au plus quelques tonnes/an. En cours de démarrage chez plusieurs oléochimistes en France, aux USA et au Japon. À côté d'usages innovants, le «CG » pourrait se développer en substitution des carbonates d'éthylène et propylène.

- Stations d'épurations: le glycérol est employé depuis plusieurs années en tant que booster de fermentation. Idem pour la méthanisation mais avec des précautions, l'accélération peut être explosive...

Nous avons ensuite souhaité aller plus loin en tentant d'être exhaustifs et de quantifier les volumes correspondant aux utilisations - réelles ou potentielles - que nous avons été en mesure d'identifier pour le glycérol (tableau 8). 
Tableau 8. Estimation de la demande mondiale en glycérine 2005-2008 en milliers de tonnes.

\begin{tabular}{|c|c|c|c|}
\hline \multirow[b]{2}{*}{2005} & 2006 & 2007 & 2008 \\
\hline & & & \\
\hline Impact du taux de croissance assumé de 3,75 \% p.a. & 1073 & 1114 & 1155 \\
\hline $\begin{array}{l}\text { Substitution du sorbitol dans les dentifrices } \\
\text { Marché du sorbitol : } 1 \mathrm{M} \text { t en } 2004 \text {, dont } 75 \% \text { sont potentiellement substituables par la glycérine }\end{array}$ & 10 & 20 & 40 \\
\hline $\begin{array}{l}\text { Substitution du propylène glycol en alimentation animale } \\
\text { Substitution des mélasses en alimentation animale } \\
\text { Le marché potentiel du propylène glycol pour cet usage : en France : 5-10 } 000 \text { tpa, dans le monde : } 100000 \text { tpa. } \\
\text { Les mélasses sont des sous-produits de l'industrie sucrière et qui présentent une valeur énergétique de loin plus élevée que celle du glycérol. }\end{array}$ & $\begin{array}{l}10 \\
-\end{array}$ & 20 & 40 \\
\hline $\begin{array}{l}\text { Substitution de l'éthylène glycol (MEG) dans les lave-glaces. } \\
\text { Plusieurs milliers de tonnes de glycérol sont déjà utilisées pour cette application en Europe du Nord. }\end{array}$ & 5 & 10 & 20 \\
\hline $\begin{array}{l}\text { Substitution de l'éthylène glycol dans les antigels } \\
\text { Substitution de l'éthylène glycol dans d'autres circuits de refroidissement. } \\
\text { La substitution du MEG par la glycérine dans les antigels a été explorée par de grands constructeurs automobiles en Europe. } \\
\text { Les quelques difficultés techniques apparues devraient être résolues sous peu. Le marché européen est estimé à } 400000 \text { tpa. }\end{array}$ & - & $\begin{array}{c}20 \\
5\end{array}$ & $\begin{array}{l}40 \\
10\end{array}$ \\
\hline $\begin{array}{l}\text { Substitution du propylène glycol dans les détergents } \\
\text { Nous considérons que le glycérol à déjà remplacé le propylène glycol dans cette application (heavy duty liquid detergents). }\end{array}$ & - & - & - \\
\hline $\begin{array}{l}\text { Glycérine contre le verglas sur les héliports, tarmacs, aéroports, etc. } \\
\text { Il y a ici de nombreuses barrières pour utiliser la glycérine. Cela tient aux sévères homologations de ce qui touche les secteurs de l'aéronautique. }\end{array}$ & - & - & 10 \\
\hline $\begin{array}{l}\text { Déshydratation du gaz. } \\
\text { Utilisé par le passé pour le gaz d'éclairage, le glycérol est désormais évalué pour la déshydratation «à la source » du gaz naturel. } \\
\text { Les volumes concernés n'ont pas été identifiés. }\end{array}$ & - & - & - \\
\hline $\begin{array}{l}\text { Agent antigel pour les ciments et bétons. } \\
\text { La glycérine abaissant leur point de congélation en hiver. }\end{array}$ & 10 & 20 & 25 \\
\hline $\begin{array}{l}\text { Agents anti-poussières } \\
\text { La glycérine peut entrer dans la formulation d'agents anti-poussières. Sont concernés : le charbon, les farines de bois, la silice, etc. }\end{array}$ & 10 & 20 & 25 \\
\hline $\begin{array}{l}\text { Stations d'épuration } \\
\text { Le glycérol confère un regain d'activité aux bactéries qui y sont utilisées. }\end{array}$ & 5 & 5 & 10 \\
\hline $\begin{array}{l}\text { Usages additionnels pour les savons, les cosmétiques et les aliments } \\
\text { Les vertus reconnues de la glycérine lui ont permis de se développer largement dans ces usages. On peut citer l'incorporation accrue par } \\
\text { Unilever de glycérine dans les savons. }\end{array}$ & 10 & 10 & 20 \\
\hline $\begin{array}{l}\text { Utilisation de la glycérine en combustion } \\
\text { en méthanisation } \\
\text { La combustion de la glycérine pose des problèmes techniques, incluant son faible pouvoir calorifique et son point de flamme élevé, mais ce sont } \\
\text { par ailleurs des avantages pour certaines applications spécifiques. Il en est de même pour la production d'électricité à partir d'unités de } \\
\text { méthanisation. }\end{array}$ & $\begin{array}{l}25 \\
10\end{array}$ & $\begin{array}{l}50 \\
13\end{array}$ & $\begin{array}{l}75 \\
17\end{array}$ \\
\hline $\begin{array}{l}\text { Conversion en épichlorhydrine } \\
\text { Solvay a annoncé la construction d'un pilote de } 10000 \mathrm{mt} \text { p.a. en France pour produire de l'épichlorhydrine à partir de glycérine. } \\
\text { Le marché mondial de l'épichlorhydrine est d'environ } 1 \mathrm{M} \mathrm{mt} \mathrm{p.a.}\end{array}$ & - & 20 & 30 \\
\hline $\begin{array}{l}\text { Chimie des C3 : « acryliques » et acroléine. } \\
\text { Avec son squelette à trois carbones, le permettrait d'accéder à ces commodités. Des développements pilote sont actuellement en cours. } \\
\text { Le marché mondial est ici de } 3 \mathrm{M} \mathrm{mt} \mathrm{p.a.}\end{array}$ & 10 & 30 & 60 \\
\hline $\begin{array}{l}\text { Production de glycols et de propylène } \\
\text { Il est connu que le glycérol peut être converti en glycols, propylène et propane. ADM a annoncé en novembre } 2005 \text { d'une unité de } \\
\text { transformation des "renewable carbohydrate-based and/or glycerol-based feedstocks" pour aller vers ces commodités. Cependant, } \\
\text { les investissements s'avèrent très élevés et les procédés "énergivores". }\end{array}$ & - & - & - \\
\hline $\begin{array}{l}\text { Augmentation de la substitution des polyols/glycols en Chine } \\
\text { La Chine a importé } 50000 \mathrm{mt} \text { supplémentaires de glycérine en } 2005 \text { par rapport à 2004. Une grande part de ces imports est destinée à } \\
\text { substituer les divers polyols/glycols largement utilisés dans ce pays. }\end{array}$ & 30 & 60 & 90 \\
\hline Substitution des carbonates d'éthylène et de propylène par le carbonate de glycérol & - & - & - \\
\hline TOTAL & 1208 & 1417 & 1667 \\
\hline
\end{tabular}


Tableau 9

\begin{tabular}{|lrrr|}
\hline Glycérol & $\mathbf{2 0 0 5}$ & $\mathbf{2 0 0 6}$ & $\mathbf{2 0 0 8}$ \\
\hline Offre kt & 1035 & 1073 & 1475 \\
Demande & 1035 & 1208 & 1667 \\
Déficit kt & 0 & 135 & 192 \\
\hline
\end{tabular}

Tableau 10. Prix prévisionnels de la glycérine à court, moyen et long terme 2006-2008.

\begin{tabular}{|lccc|}
\hline Pays & Mid 2006 & Fin 2006 & 2008 \\
\hline USA & & & \\
$99,5 \%$ Kasher, prix « spot » & USD 0,34 c/lb & USD 0,30 c/lb & USD 0,40 c/lb \\
$99,5 \%$ origine suif, prix & USD 0,29 c/lb & USD 0,28 c/lb & USD 0,36 c/lb \\
« spot » & & & \\
Europe & & & \\
$99,5 \%$ Kasher, prix « spot » & EUR $430 \mathrm{pmt}$ & EUR $425 \mathrm{pmt}$ & EUR $500 \mathrm{pmt}$ \\
$99,5 \%$ origine suif, prix & EUR $400 \mathrm{pmt}$ & EUR $400 \mathrm{pmt}$ & EUR 475 pmt \\
« spot » & EUR $80 \mathrm{pmt}$ & EUR $60 \mathrm{pmt}$ & EUR 150 pmt \\
Qualité brute base $80 \%$ & & &
\end{tabular}

Prix « rendu-vrac ». Taux de change pour la qualité raffinée: 1 EUR = 1,19 USD.

\section{Conclusion}

Ainsi, à partir de scénarios pondérés, nous arrivons à la conclusion que la perspective que la demande dépasse l'offre n'est pas aberrante. Cela peut se résumer de la façon suivante (tableau 9) :

Demande $2006=$ demande $2005 \times 1,0375+\Delta$ $(2006-2005)=1073+135=1208 \mathrm{kt}$

Demande $2008=$ demande $2005 \times[1,0375]^{3}$ $+\Delta(2008-2007)=1155+512=1667 \mathrm{kt}$

Ce déficit de 192 kt pourrait écouler les stocks et permettre aux prix de se raffermir à la mi-2008 et même plus tôt. D'ici là, les prix vont rester peu élevés et risquent même de baisser encore. Quant aux prévisions à court, moyen et long terme, elles sont résumées dans le tableau (tableau 10, MH/SC, 15/03/2006). 